\title{
Upregulation of TREM2 Ameliorates Neuroinflammatory Responses and Improves Cognitive Deficits Triggered by Surgical Trauma in Appswe/PS1dE9 Mice
}

\author{
Yanhua Jiang ${ }^{\mathrm{a}}$ Zhe Lia $\mathrm{Hong} \mathrm{Ma}^{\mathrm{a}}$ Xuezhao Cao ${ }^{\mathrm{a}}$ Fang Liu \\ Ayong Tian ${ }^{\mathrm{a}}$ Xijia Sun ${ }^{\mathrm{a}}$ Xiaoqian $\mathrm{Li}^{\mathrm{a}}$ Jun Wang ${ }^{\mathrm{a}}$
}

${ }^{a}$ Department of Anesthesiology, the First Hospital of China Medical University, Shenyang, ${ }^{b}$ Department of Neurology, the First Hospital of China Medical University, Shenyang, China

\section{Key Words}

Trem2 • Microglia • Cytokines • Hippocampus • Neuroinflammation • Postoperative Cognitive Dysfunction $(\mathrm{POCD})$

\begin{abstract}
Background/Aims: TREM2 plays a crucial role in modulating microglial function through interaction with DAP12, the adapter for TREM2. Emerging evidence has demonstrated that TREM2 could suppress neuroinflammatory responses by repression of microglia-mediated cytokine production. This study investigated the potential role of TREM2 in surgery-induced cognitive deficits and neuroinflammatory responses in wild-type (WT) and APPswe/PS1dE9 mice. Methods: Adult APPswe/PS1dE9 transgenic male mice (a classic transgenic model of Alzheimer's disease, 3 months old) and their age-matched WT mice received intracerebral lentiviral particles encoding the mouse TREM2 gene and then were subjected to partial hepatectomy at 1 month after the lentiviral particle injection. The behavioral changes were evaluated with an open-field test and Morris water maze test on postoperative days 3, 7, and 14. Hippocampal TREM2, DAP12, and interleukin (IL)-1 $\beta$ were measured at each time point. Ionized calcium-binding adapter molecule 1 (Iba-1), microglial M2 phenotype marker Arg1,

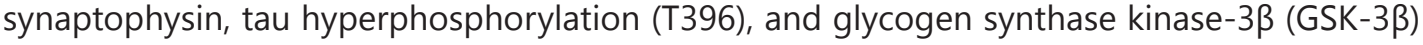
were also examined in the hippocampus. Results: Surgical trauma induced an exacerbated cognitive impairment and enhanced hippocampal IL-1 $\beta$ expression in the transgenic mice on postoperative days 3 and 7. A corresponding decline in the levels of TREM2 was also found on postoperative days 3,7 , and 14. Overexpression of TREM2 downregulated the levels of IL-1 $\beta$, ameliorated T396 expression, inhibited the activity of GSK-3 $\beta$, and improved sickness behavior. Increased Arg1 expression and a high level of synaptophysin were also observed in the transgenic mice following TREM2 overexpression. Conclusion: The downregulation of TREM2 exacerbated surgery-induced cognitive deficits and exaggerated neuroinflammatory responses in this rodent model. Overexpression of TREM2 potentially attenuated these effects by decreasing the associated production of proinflammatory cytokines, inhibiting tau hyperphosphorylation, and enhancing synaptophysin expression.




\section{Cellular Physiology Cell Physiol Biochem 2018;46:1398-1411 \\ \begin{tabular}{l|l} 
DOI: 10.1159/000489155 & Ond 2018 The Author(s). Published by S. Karger AG, Basel \\
www.karger.com/cpb
\end{tabular}}

Jiang et al.: TREM2 Ameliorates Postoperative Cognitive Deficits

\section{Introduction}

Postoperative cognitive dysfunction (POCD) is characterized by the progressive deterioration of intellectual/cognitive function following both cardiac and major noncardiac surgery $[1,2]$. The International Study of POCD estimated the overall incidence of POCD at $25.8 \%$ at 1 week and $9.9 \%$ after 3 months [1]. POCD is associated with prolonged hospitalization, delayed recovery, and an increased risk of disability and mortality [3]. Therefore, it is crucial to identify underlying mechanisms of POCD in order to treat or prevent cognitive impairments after surgery.

Proinflammatory cytokines in the brain reduce neurogenesis and inhibit neuronal functions, including long-term potentiation (LTP) and dendritic branching, which are involved in memory formation and maintenance [4]. Mounting evidence indicates that POCD is associated with surgery-induced neuroinflammatory processes, which may influence neuronal function either directly or through modulation of intraneuronal pathways [5-7]. High levels of neuroinflammatory cytokines, such as interleukin (IL)-1 $\beta$, IL-6, and tumor necrosis factor (TNF)- $\alpha$ play a pivotal role in surgery-induced cognitive deficits $[2,8]$. Inhibition of central proinflammatory cytokine signaling restores neuronal function and reverses cognitive deficits induced by chronic neuroinflammation [9].

TREM2 plays a crucial role in modulating microglial function through interaction with DAP12 to promote microglial phagocytosis [10] and survival [11]. As a microglial immune receptor, TREM2 was revealed to regulate inflammatory responses mediated by microglia [12]. Mounting evidence has indicated that TREM2 inhibits microglia-mediated production of proinflammatory cytokines induced by lipopolysaccharide (LPS) or apoptotic cells [13, 14]. A study by Jiang et al. revealed that knockdown of TREM 2 on microglia exacerbated neuroinflammation in SAMP8 mice, an animal model of senescence [15]. More importantly, a recent study showed that selectively overexpressed TREM2 on microglia markedly ameliorated $A \beta$-induced neuroinflammation [16]. These data indicate that TREM2 is critical in maintaining homeostasis of the central nervous system.

The severity of the surgery influences the magnitude of the immune response and has been shown to correlate with the degree of postoperative inflammation and sickness behavior $[17,18]$. Canet et al. showed that the incidence of POCD in elderly patients after minor surgery (primarily laparoscopy) was significantly lower than that after cardiac and noncardiac major surgery, suggesting that extent of surgery contributes to postoperative brain dysfunction [19]. Additionally, Rosczyk et al. have demonstrated that locomotor activity is not depressed in either adult or aged mice following sham operation (minor abdominal surgery), which reveals that the decrease in locomotion is not due to minor surgical procedures [20]. It is likely that a more "major" surgery (partial hepatectomy) would induce a state of neuroinflammation that could result in sickness behavior [2, 7, 21-25].

Our previous study revealed that surgery-induced proinflammatory cytokines in the brain of aged rats play an important role in the development of POCD [2, 7, 21]. These earlier findings suggest that neuroinflammatory response may be an important underlying mechanism for POCD. Activation of TREM2 was revealed to regulate microglial function including phagocytosis and cytokine production [26,27]. Central nervous system dysfunction attendant with Alzheimer's disease (AD) may increase the risk for the development of POCD [28]. The present study focuses on the role of TREM2 in surgery-induced microglial activation, neuroinflammatory responses, and cognitive impairment in APPswe/PS1dE9 mice, a classic transgenic model of AD. We sought to determine whether TREM2 overexpression in the brain ameliorates surgery-induced neuroinflammatory responses by modulating microglial function and improved neurobehavioral deficits.

\section{Materials and Methods}

Animals

Adult APPswe/PS1dE9 transgenic male mice (3 months old) were randomly divided into four groups: control group ( $n=10)$, surgery group $(n=30)$, surgery+vector group $(n=30)$, and surgery+TREM2 group $(n$ 


\section{Cellular Physiology Cell Physiol Biochem 2018;46:1398-1411 \begin{tabular}{l|l} 
and Biochemistry Published $1159 / 000489155$ & $\begin{array}{l}\text { D) } 2018 \text { The Author(s). Published by S. Karger AG, Basel } \\
\text { www.karger.com/cpb }\end{array}$ \\
\hline
\end{tabular}}

Jiang et al.: TREM2 Ameliorates Postoperative Cognitive Deficits

= 30). In parallel, age-matched wild-type (WT) mice were divided into the same four treatment categories, for a total of eight groups. It is noteworthy that there was no amyloid neuropathology and spatial cognitive impairments in the 3-month-old APPswe/PS1dE9 mice. All animals were housed in a standard animal room on a 12-hour light/dark cycle with ad libitum access to food and water. The control mice stayed in their home cage. Partial hepatectomy was performed under general anesthesia (a gas mixture of 1.5\%-2.0\% isoflurane and oxygen) in all surgery groups. Briefly, the liver was exposed through a 1- to 2-cm midline abdominal incision. The left lateral lobes of the liver (approximately corresponding to $30 \%$ of the organ) were excised. The wound was then infiltrated with $0.25 \%$ bupivacaine. The mice in the surgery+TREM2 group received an intracerebroventricular injection of adeno-associated virus particles encoding the mouse TREM2 gene. The mice in the surgery+vector group received an intracerebroventricular injection of a control adeno-associated virus vector. As intracerebral adeno-associated virus particle injection is a surgical intervention, which could inadvertently produce memory impairments, the mice in the surgery group received a similar injection of vehicle in the same place. All procedures were conducted in accordance with the National Institutes of Health Guide for Care and Use of Laboratory Animals and approved by the China Medical University Animal Care and Use Committee.

\section{Experimental procedure}

The mice received an intracerebroventricular injection of adeno-associated virus particles encoding the mouse TREM2 gene. All mice were trained in a Morris water maze (MWM) with 3 trials per day for 6 consecutive days before surgery. Partial hepatectomy was performed under general anesthesia in all surgery groups at 1 month after the lentiviral particle injection. The behavioral changes were evaluated with an open-field test and MWM test on postoperative days 3, 7, and 14. The mice were sacrificed following the behavioral tests at each time point ( $n=10$ per day) and the hippocampi were rapidly harvested for biochemical analysis and stored at $-80^{\circ} \mathrm{C}$ until processed $(n=15$ for Western blot and $n=15$ for real-time PCR).

\section{Open-field test}

An open-field test was performed to assess exploratory activity and anxiety-like behavior on postoperative days 3,7 , and 14 . The mice were placed individually into the center of the area and allowed a 5 -min free exploration period in a square wooden arena $(100 \times 100 \times 50 \mathrm{~cm})$. The test was conducted in a quiet room in the morning (8:00-12:00 a.m.) by an investigator who was blinded to the intervention. Spontaneous locomotor activity was assessed by the total amount of distance traveled in the chamber. The time in the central area was taken as measures of anxiety and exploratory behavior.

\section{Morris water maze}

Spatial learning and memory was evaluated using an MWM test. Briefly, the mice were placed on the platform for $30 \mathrm{~s}$ before the start of each trial and released into the water from one of four randomly assigned release points (N, W, S, and E). In all trials, mice were allowed to swim until they landed on the hidden platform. If a mouse failed to find the platform within $60 \mathrm{~s}$, it was gently guided toward the platform and left there for $15 \mathrm{~s}$. On postoperative days 3,7 , and 14, all mice were subjected to a probe trial in which the platform was removed and each animal freely searched the pool for the platform for $60 \mathrm{~s}$. The time spent in the target quadrant was taken as a measure of spatial memory. The MWM test was performed in a blind manner (the surgical incision area was covered with tape for all groups).

\section{Adeno-associated virus vector preparation}

Adeno-associated virus vectors encoding the mouse TREM2 gene (NCBI ID: NM_031254.3) and a control adeno-associated virus vector were provided by Genechem Co. (Shanghai, China). The following short hairpin sequences (TREM2 shRNA: $5^{\prime}$-TGATGCTGGAGATCTCTGGGTTCAAGAGACCCAGAGATCTCCAGCATCTTTTTTC- $3^{\prime}$ and control shRNA: $5^{\prime}$-TGATGCTGAAGGTCGCTTGGTTCAAGAGACCAAGCGACCTCCAGCATCTTTTTTC- $3^{\prime}$ ) were synthesized and subcloned into the adeno-associated virus vectors respectively. The adeno-associated virus vectors were purified and then co-transfected with packaging vectors into 293FT cells. The supernatant was collected after $48 \mathrm{~h}$, and the lentiviral particles in the supernatant were concentrated at 1:100 by ultracentrifugation and recovered by suspension in PBS. Viral titers were determined by a commercial titration ELISA kit (TaKaRa; titer for adeno-associated virus particles containing TREM2 vector: $2.08 \times 10^{15} \mathrm{TU} / \mu \mathrm{l}$; titer for control adeno-associated virus particles: $5.28 \times 10^{15} \mathrm{TU} / \mu \mathrm{l}$ ). 


\section{Cellular Physiology Cell Physiol Biochem 2018;46:1398-1411 and Biochemistry Published online: April 23, $2018 \quad \begin{aligned} & \text { DOI: 10.1159/000489155 } 2018 \text { The Author(s). Published by S. Karger AG, Basel } \\ & \text { www.karger.com/cpb }\end{aligned}$}

Jiang et al.: TREM2 Ameliorates Postoperative Cognitive Deficits

Intracerebral lentiviral particle injection

Three-month-old APPswe/PS1dE9 mice and their age-matched WT mice were anesthetized with isoflurane and fixed on a stereotactic frame. Injection of adeno-associated virus particles was performed in the hippocampus (one deposit) of each hemisphere with $1 \mu$ l adeno-associated virus particles using a micropipette attached to a 10- $\mu$ l Hamilton syringe. Stereotactic coordinates of injection sites from bregma were for hippocampus: anteroposterior $=-2$; mediolateral $=1.2$; dorsoventral $=-2 \mathrm{~mm}$. One month later, the therapeutic effects of adeno-associated virus vector-mediated TREM2 overexpression were verified.

\section{Western blot analysis}

Brain tissues were homogenized in ice-cold lysis buffer containing complete protease inhibitor cocktail. The lysates were shaken in an ice bath for $30 \mathrm{~min}$ and cleared by centrifugation $\left(12,000 \mathrm{~g}\right.$ for $\left.10 \mathrm{~min}, 4^{\circ} \mathrm{C}\right)$. The supernatant was collected followed by immunoblot analysis. The proteins $(60 \mu \mathrm{g})$ were transferred to polyvinylidene fluoride microporous membranes. Then, the membranes were blocked with $5 \%$ fat-free milk at room temperature for $2 \mathrm{~h}$ and incubated overnight at $4^{\circ} \mathrm{C}$ with the following primary antibodies: rabbit monoclonal anti-IL-1 $\beta$ (1:2, 000; Abcam, Cambridge, UK), rabbit anti-synaptophysin (1:20, 000; Abcam), rabbit anti-ionized calcium-binding adapter molecule 1 (Iba-1) (1:800; Abcam), rabbit anti-phosphorMAPT-T396 polyclonal (1:1, 000; ABclonal, Wuhan, China), rabbit anti-GSK-3 $\beta$ polyclonal $(1: 1,000$; ABclonal), or polyclonal anti- $\beta$-actin (1:4, 000; ZSGB-BIO, Beijing, China). After washing with TBST three times, the membranes were incubated with horseradish peroxidase-coupled secondary antibody $(1: 5,000)$ for $2 \mathrm{~h}$ at room temperature. The immunoreactivity was detected using enhanced chemiluminescence (ECL Advance Kit; Amersham Biosciences, USA) and visualized using Image Lab software (Bio-Rad Laboratories, Hercules, CA). The relative expression levels of protein were normalized by the ratio of target protein to $\beta$-actin.

\section{Real-time PCR}

Total RNA was extracted by TRIzol reagent (Takara, Otsu, Japan) according to the manufacturer's instructions. RNA $(1.0 \mu \mathrm{g})$ for each condition was reverse transcribed using the PrimeScript RT reagent Kit with gDNA Eraser (Takara). Two microliters of cDNA was used to perform quantitative real-time PCR. The following primers were used to amplify the mRNA: TREM2: 5'-AGAAGGGGAATGGGAGC-3' (forward) and 5'-GAGGTGGGTGGGAAGGA-3' (reverse), Arg1: 5'-AGAAGCGGAATGGGAGC-3' (forward) and 5'-GAGGTGGGTGGGAAGGA-3' (reverse). Glyceraldehyde 3-phosphate dehydrogenase (GAPDH) was used as an internal control. Primers were obtained from Sangon Biotech (China). The reverse transcription reaction was carried out under the following conditions: $95^{\circ} \mathrm{C}$ for $10 \mathrm{~min}$ (initial denaturation); followed by 40 cycles of $95^{\circ} \mathrm{C}$ for $20 \mathrm{~s}, 62^{\circ} \mathrm{C}$ for $30 \mathrm{~s}$ and $72^{\circ} \mathrm{C}$ for $30 \mathrm{~s}$ (amplification). The relative gene expression was determined by calculating the expression ratio of the gene of interest to GAPDH. The relative expression of mRNA was quantified using the $2-\Delta \Delta$ Ct method.

\section{Results}

Intracerebral injection of the adeno-associated virus containing TREM2 cDNA effectively increased the TREM2 mRNA levels

The TREM2 mRNA levels in the hippocampus of the APPswe/PS1dE9 mice were 3.5fold higher than those in the gene-matched controls $(P<0.001)$ at 1 month after injection of adeno-associated virus particles (Fig. 1). Similarly, the levels of TREM2 in the hippocampus of the WT mice were elevated by 3.1-fold $(P<0.001)$ (Fig. 1). Note that no significant difference was observed in the mRNA levels of DAP12, the adapter for TREM2, in the brain of both WT and APPswe/PS1dE9 mice at the same time point (data not shown).

Surgical trauma decreased the TREM2 mRNA levels in both WT and transgenic mice

Surgical trauma significantly decreased the TREM2 mRNA levels in the hippocampus of APPswe/PS1dE9 mice on postoperative days $3(P<0.001), 7(P<0.001)$, and $14(P<0.001)$ (Fig. 2). Similar findings were observed for TREM2 following surgical procedure in the WT mice on postoperative days $3(P<0.001)$ and $7(P<0.001)$ (Fig. 2$)$. Taken together, these 


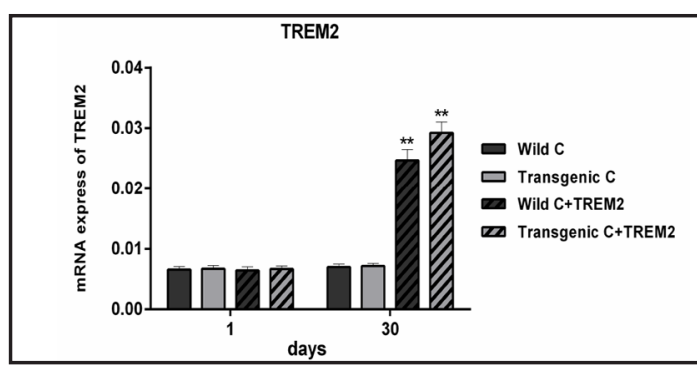

Fig. 1. Intracerebral injection of adeno-associated virus containing TREM2 cDNA effectively increased the mRNA levels of TREM2 in both wild-type (WT) and transgenic mice. The results are represented as the mean $\pm \mathrm{SEM}$. ${ }^{* *} \mathrm{P}<0.001$ versus the genematched control group.

findings indicated that the downregulation of TREM2 was a direct response to the surgical challenge. Surgical trauma and exogenous TREM2 failed to alter the activity of PP2A in the brain of both WT and APPswe/PS1dE9 mice.

Surgical trauma induced behavioral deficits and TREM2 overexpression improved cognitive impairment in APPswe/PS1dE9 mice

Surgical trauma significantly decreased the total distance travelled in the WT mice compared with the gene-matched controls in the open-field test on postoperative day 3 $(P<0.05)$ and recovered on day 7 (Fig. 3A). In the transgenic mice, the same impairments were observed on postoperative day $3(P$ $<0.001)$, persisted until day $7(P<0.05)$, and recovered on day 14 (Fig. 3A). The center duration of the transgenic surgery mice was significantly shorter compared with that of gene-matched controls on postoperative days $3(P<0.001)$ and $7(P$ $=0.002$ ) (Fig. 3B). Similarly, a significant difference for the center duration was also observed between the WT surgery mice and the controls on postoperative day $3(P=0.003)$ and recovered on day 7 (Fig. 3B). Compared with the day-matched WT surgery mice, additional decreases in the total distance travelled and the center duration were found in the transgenic surgery mice on postoperative day 7 ( $P$ $<0.05$ and $P<0.05$, respectively) (Fig. 3). TREM2 overexpression reversed the total distance travelled and the center duration in the transgenic surgery mice on

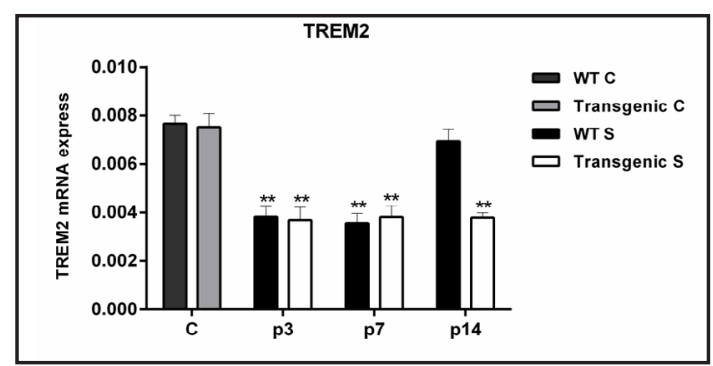

Fig. 2. Surgical trauma significantly decreased TREM2 expression in both wild-type (WT) and APPswe/PS1dE9 mice. The results are represented as the mean \pm SEM. ${ }^{* *} \mathrm{P}<0.001$ versus the gene-matched control group. C: control group; S: surgery group; P3, P7, and P14: postoperative days 3, 7, and 14, respectively.

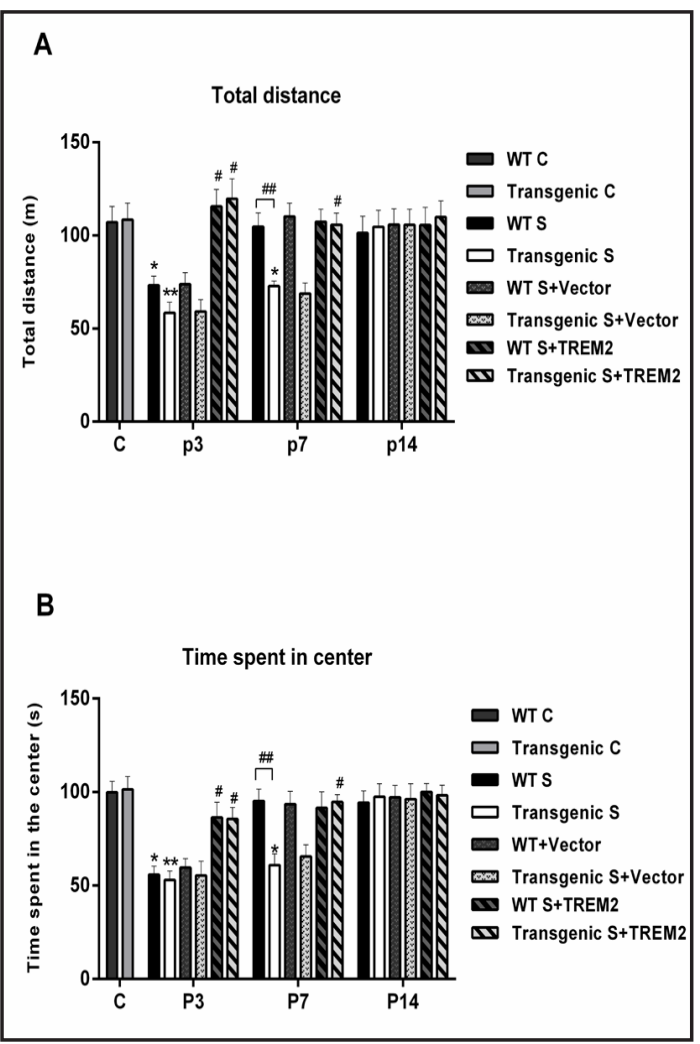

Fig. 3. Surgical trauma significantly decreased the total distance travelled and the center duration in both wild-type (WT) and transgenic mice in the open-field test. TREM2 overexpression reversed surgery-induced sickness behavior in both WT and transgenic surgery mice. (A) Total distance moved in the chamber. (B) The time in the central area. The results are represented as the mean $\pm \mathrm{SEM} .{ }^{*} \mathrm{P}<0.05$, ${ }^{* *} \mathrm{P}<0.001$ versus the gene-matched control group; ${ }^{\#} \mathrm{P}<0.05$ versus the gene-matched surgery group. ${ }^{\# \#} \mathrm{P}<0.05$ versus the day-matched WT surgery group. C: control group; S: surgery group; P3, P7, and P14: postoperative days 3,7 , and 14 , respectively. 
postoperative days $3(P<0.001$ and $P=$ 0.016 , respectively) and $7(P=0.046$ and $P=0.012$, respectively) (Fig. 3). Similar changes were observed in the WT mice on postoperative day $3(P=0.007$ and $P=$ 0.026 , respectively).

Both WT and APPswe/PS1dE9 mice showed improvements in swimming data over 6 consecutive training days in the MWM test. However, the WT mice performed significantly better (swam less distance and with less latency) than APPswe/PS1dE9 mice on days $2(P=0.033$ and $P=0.01$, respectively) and $3(P=0.01$ and $P=0.02$, respectively) (Fig. 4). Surgical trauma significantly decreased the time spent in the target zone during the probe trial in the WT mice compared with the gene-matched controls on postoperative day $3(P<0.001)$ and recovered on day $7(P>0.05)$ (Fig. 5A). However, in the transgenic mice, the shorter time spent in the target zone was maintained until day $7(P=0.02)$ and recovered on day $14(P=0.99)$ (Fig. 5A). A significant difference was found for the time spent in the target zone between the WT and transgenic surgery mice on postoperative day $7(P=0.03)$. Treatment with TREM2 significantly improved spatial learning impairment in the APPswe/PS1dE9 mice on postoperative day $7(P=0.007)$ (Fig. $5 A)$. Overexpression of TREM2 failed to significantly affect spatial learning abilities in the WT mice on postoperative day $3(P=0.97)$ (Fig. 5A). It should be noted that no significant difference in swimming speed was observed following injection of adeno-associated virus or overexpression of TREM2 between the WT and APPswe/PS1dE9 mice (Fig. 5B).

\section{Overexpression of TREM2 directly ameliorated surgery-induced neuroinflammatory responses \\ Surgical trauma upregulated IL-} $1 \beta$ expression in the WT mice on postoperative days 3 and $7(P<0.001$ and $P<0.001$, respectively) and recovered on day $14(P=0.092)$. The protein levels of IL-1 $\beta$ in the transgenic mice were significantly increased compared with the gene-matched controls on postoperative days $3(P<0.001)$ and $7(P<0.001)$ (Fig.

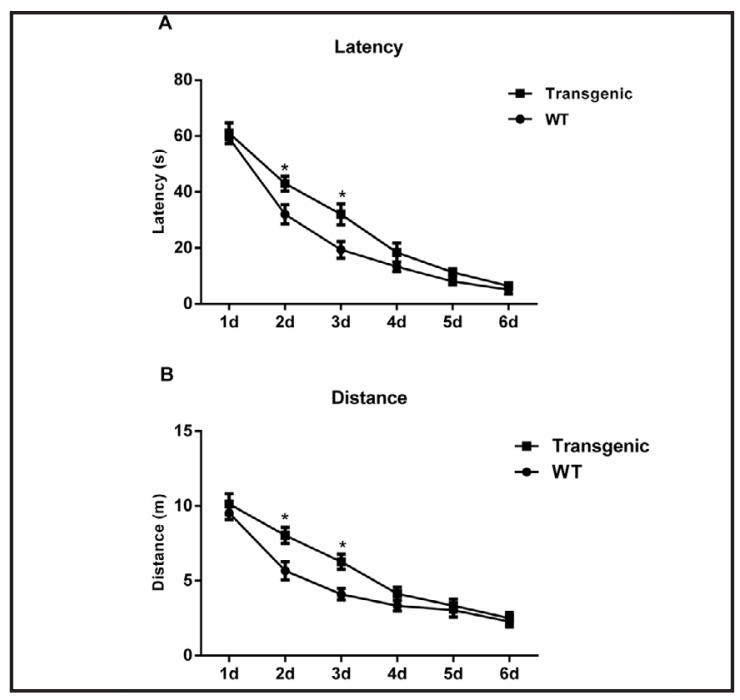

Fig. 4. The wild-type (WT) mice performed significantly better (swam less distance and with less latency) than APPswe/PS1dE9 mice on training days 2 and 3 in a Morris water maze test. ${ }^{*} \mathrm{P}<0.05$ versus the daymatched transgenic mice.

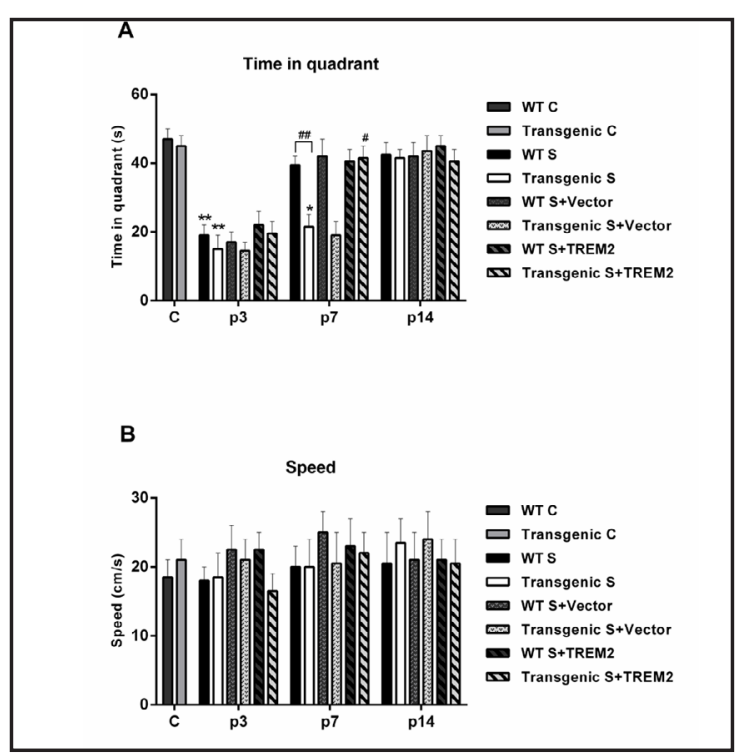

Fig. 5. Surgical trauma exacerbated spatial learning and memory impairment during the probe trial in both wild-type (WT) and transgenic mice. A significant difference was found for the time spent in the target zone between the WT and transgenic surgery mice on postoperative day 7. Treatment with TREM2 significantly improved sickness behavior in the transgenic mice on postoperative day 7 . The results are represented as the mean $\pm \mathrm{SEM} .{ }^{*} \mathrm{P}<0.05,{ }^{* *} \mathrm{P}<0.001$ versus the gene-matched control group; $\mathrm{P}<0.05$ versus the genematched surgery group. ${ }^{\# \# P<0.05}$ versus day-matched WT surgery group. C: control group; S: surgery group; P3, P7, and P14: postoperative days 3,7 , and 14 , respectively. 
6). Compared with the day-matched WT surgery group, the protein levels of IL- $1 \beta$ were significantly higher in the transgenic surgery mice on postoperative day $7(P<$ 0.05). Treatment with TREM 2 remarkably attenuated surgery-induced upregulation of IL-1 $\beta$ in both WT and transgenic mice on postoperative day $7(P<0.05$ and $P<0.05$, respectively) (Fig. 6). Vector alone failed to alter the levels of IL-1 $\beta$ in both WT and transgenic mice at any time point.

Surgical trauma enhanced microglial activation, and treatment with TREM2 decreased the levels of Iba-1

Surgical trauma significantly increased the levels of Iba-1 in both WT and transgenic mice compared with the gene-matched controls on postoperative day $3(P<0.05)$ (Fig. 7). Although Iba-1 expression in the WT subjects returned to baseline on day $7(P$ $=0.99$ ), the levels of Iba-1 in the transgenic subjects remained significantly upregulated on postoperative day $7(P=0.002)$ and recovered on day $14(P=0.99)$ (Fig. 7). The levels of Iba-1 were significant higher in the transgenic surgery mice compared with the day-matched WT counterparts on postoperative day $7(P<0.05)$. Treatment with TREM2 reduced the levels of Iba- 1 in the APPswe/PS1dE9 mice compared with that of the controls on postoperative days 3 $(P=0.034)$ and $7(P=0.025)$ (Fig. 7). Vector alone failed to alter the levels of IL- $1 \beta$ in both WT and transgenic mice at any time point.

Surgical trauma significantly
downregulated the mRNA levels of
M2 phenotype marker Arg1 and
overexpression of TREM2 increased the
levels of Arg1
There was no significant difference for the baseline levels of M2 phenotype marker Arg1 in both WT and transgenic mice $(P=0.99)$. As shown in Fig. 8 , the Arg1 mRNA levels were significantly decreased following surgical challenge in both WT and APPswe/PS1dE9 mice on postoperative day $3(P<0.001$ and $P<0.001$, respectively). Although Arg1 mRNA expression in the WT surgery subjects returned to baseline on day $7(P=0.146)$, the levels of Arg1 in the transgenic subjects remained significantly

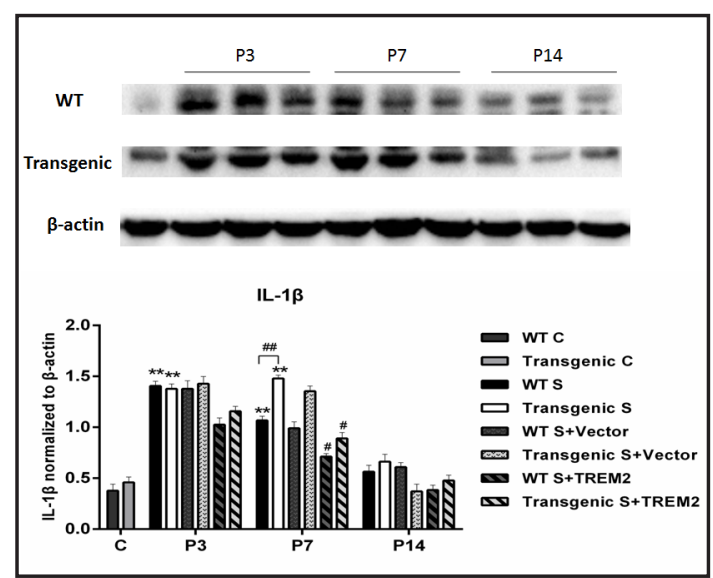

Fig. 6. Compared with the day-matched wild-type (WT) surgery group, the protein levels of IL-1 $\beta$ were significantly higher in the transgenic surgery mice on postoperative day 7. Treatment with TREM2 significantly downregulated IL-1 $\beta$ expression in the brains of both WT and transgenic surgery mice on postoperative day 7 . The results are represented as the mean \pm SEM. ${ }^{* *} \mathrm{P}<0.001$ versus the gene-matched control group $" \mathrm{P}<0.05$ versus the gene-matched surgery group ${ }^{\# \# ~} \mathrm{P}<0.05$ versus the day-matched WT surgery group. C: control group; S: surgery group; P3, P7, and P14: postoperative days 3, 7, and 14, respectively.

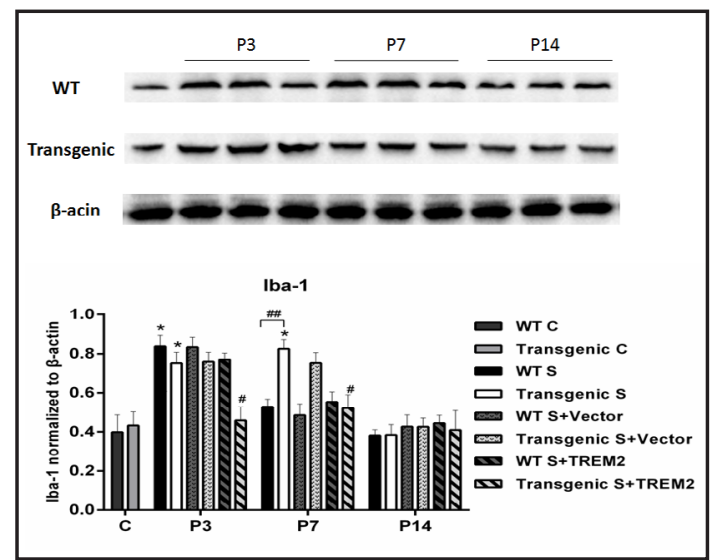

Fig. 7. Surgical trauma upregulated microglial Iba1 expression in both wild-type (WT) and transgenic mice. Treatment with TREM2 reduced the levels of Iba-1 in the APPswe/PS1dE9 mice compared with that of the controls on postoperative days 3 and 7. The results are represented as the mean \pm SEM. ${ }^{*} \mathrm{P}<0.05$ versus the gene-matched control group; ${ }^{*} \mathrm{P}<0.05$ versus the gene-matched surgery group. ${ }^{\# \#} \mathrm{P}<0.05$ versus the day-matched WT surgery group. C: control group; S: surgery group; P3, P7, and P14: postoperative days 3,7 , and 14 , respectively. 
decreased on postoperative day $7(P<0.001)$ and recovered on day $14(P>$ 0.05) (Fig. 8). Treatment with TREM2 significantly increased the Arg1 mRNA levels in the transgenic mice on postoperative days $3(P$ $=0.02)$ and $7(P=0.04)$ (Fig. 8).

Overexpression of TREM2 ameliorated surgery-induced tau hyperphosphorylation

Surgical trauma markedly increased the protein levels of T396 in the brain of APPswe/PS1dE9 mice on postoperative days $3(P<0.001), 7(P<0.001)$, and $14(P=0.025)$ (Fig. 9). The levels of T396 were significant higher in the WT surgery mice compared with that in the gene-matched controls on postoperative day $3(P<0.05)$. The levels of T396 were significant higher in the transgenic mice compared with the day-matched WT surgery mice on postoperative days 7 and $14(P<0.001$ and $P$ $<0.001$, respectively) (Fig. 9). Treatment with TREM2 significantly reduced the levels of T396 in the brain of APPswe/PS1dE9 mice following surgical challenge on postoperative days $3(P<$ 0.05), $7(P<0.05)$, and $14(P$ $<0.05$ ) (Fig. 9).

\section{Treatment with TREM2} ameliorated surgery-

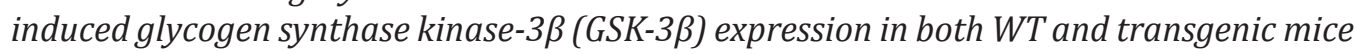
Surgical trauma significantly increased the levels of GSK-3 $\beta$ in the WT mice on postoperative day $3(P<0.05)$, which returned to baseline on day $7(P=0.86)$ (Fig. 10). GSK-3 $\beta$ activity was significantly increased following surgical challenge in the APPswe/ PS1dE9 mice on postoperative days $3(P<0.05), 7(P<0.05)$, and $14(P<0.05)$ (Fig. 10). Compared with the day-matched WT surgery mice, higher levels of GSK-3 $\beta$ were observed in the transgenic mice on postoperative days 7 and $14(P<0.05$ and $P<0.05$, respectively) (Fig. 10). Overexpression of TREM 2 markedly attenuated the activity of GSK- $3 \beta$ in the transgenic

Fig. 8. The mRNA levels of Arg1 were significantly decreased following surgical challenge in both wild-type (WT) and APPswe/PS1dE9 mice. TREM2 overexpression upregulated Arg1 expression in the transgenic mice on postoperative days 3 and 7 . The results are expressed as the mean \pm SEM. ${ }^{* *} \mathrm{P}<0.001$ versus the gene-matched control group; ${ }^{\#} \mathrm{P}<0.05$ versus the day-matched transgenic surgery group. C: control group; S: surgery group; P3, P7, and P14: postoperative days 3, 7, and 14 , respectively.

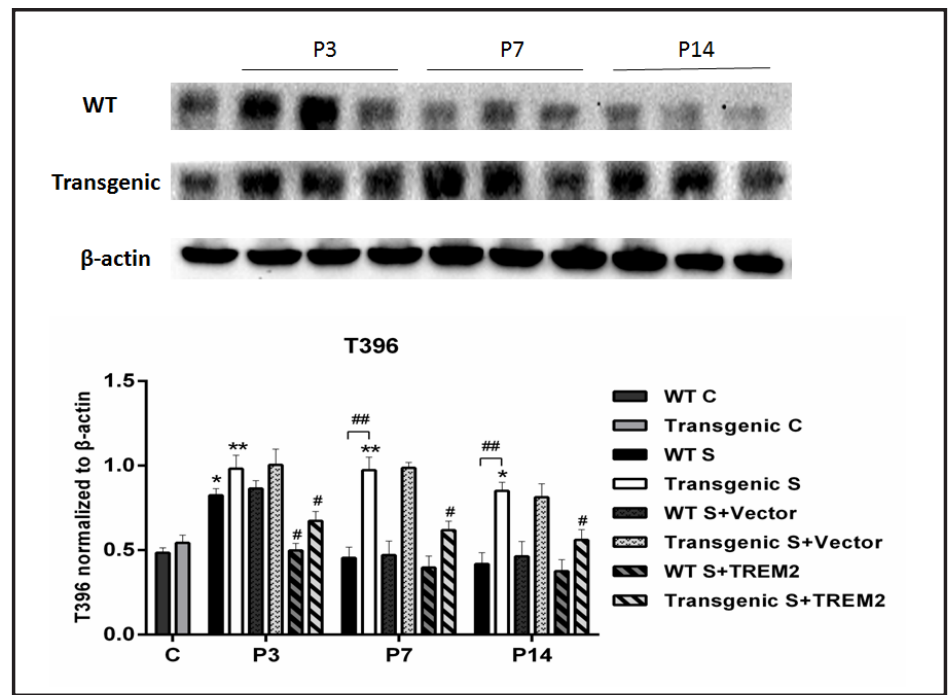

Fig. 9. Surgical trauma markedly increased the protein levels of T396 in the brain of APPswe/PS1dE9 mice on postoperative days 3, 7, and 14. Treatment with TREM2 significantly reversed surgery-induced downregulation of T396. The results are represented as the mean \pm SEM. ${ }^{*} \mathrm{P}<0.05,{ }^{* *} \mathrm{P}<0.001$ versus the gene-matched control group; ${ }^{\#} \mathrm{P}<0.05$ versus the gene-matched surgery group. ${ }^{\#} \mathrm{P}<0.001$ versus the day-matched wild-type (WT) surgery group. C: control group; S: surgery group; P3, P7, and P14: postoperative days 3, 7, and 14, respectively.

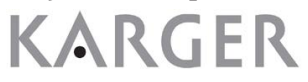


Fig. 10. Surgical trauma significantly increased the levels of GSK-3 $\beta$ in the APPswe/PS1dE9 mice on postoperative days 3,7 , and 14 . Treatment with TREM2 ameliorated surgery-induced upregulation of GSK-3 $\beta$ in both wild-type (WT) and transgenic mice. ${ }^{*} \mathrm{P}<0.05$ versus the gene-matched control group; ${ }^{\#}<<0.05$ versus the gene-matched surgery group. ${ }^{\# \#} \mathrm{P}<0.05$ versus the day-matched WT surgery group. C: control group; S: surgery group; P3, P7, and P14: postoperative days 3, 7, and 14, respectively.

mice on postoperative days $3(P<0.05), 7$ $(P<0.05)$, and $14(P<0.05)$.

Surgical trauma significantly
decreased the levels of synaptophysin,
and TREM2 overexpression
upregulated synaptophysinexpression
following the surgical procedure
There was no significant difference in basal hippocampal synaptophysin between the WT and transgenic mice $(P>$ 0.05) (Fig. 11). The levels of synaptophysin were downregulated in both WT and transgenic mice on postoperative day 3 $(P=0.041$ and $P=0.028$, respectively) and recovered on day $7(P>0.05)$ (Fig. 11). Overexpression of TREM 2 markedly increased synaptophysin expression in both WT and transgenic mice compared with the gene-matched surgery group on postoperative day $3(P<0.05$ and $P<0.05$, respectively).

\section{Discussion}
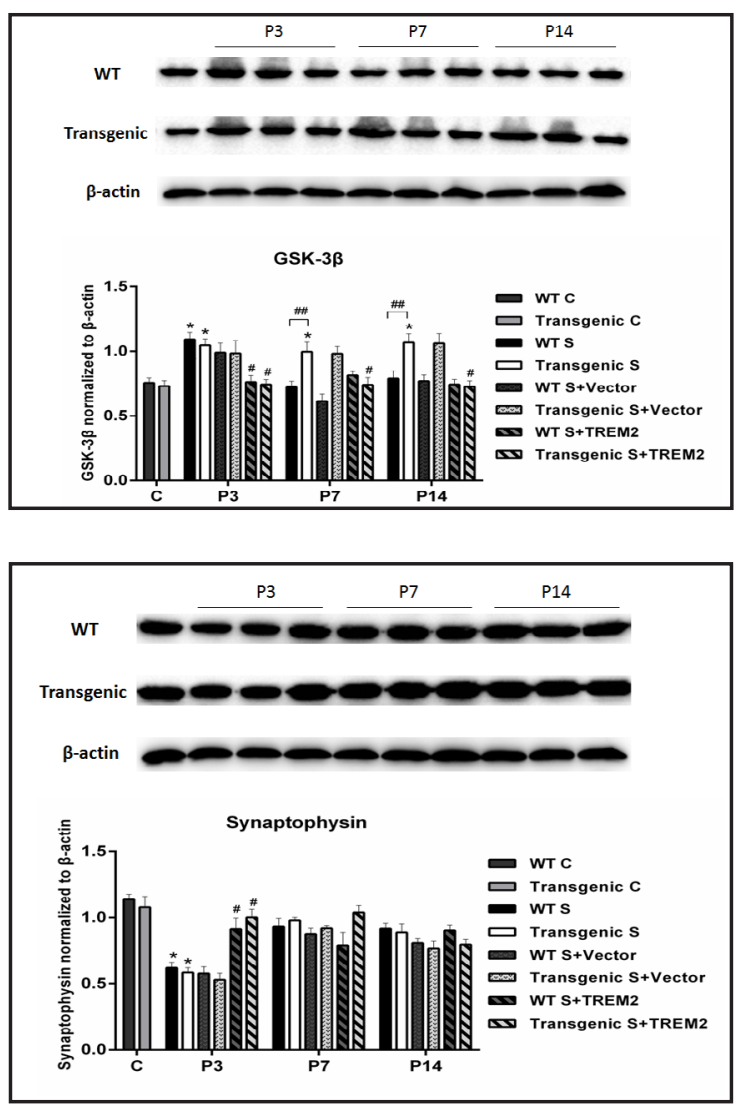

Fig. 11. Surgical trauma significantly decreased the levels of synaptophysin in both wild-type (WT) and transgenic mice on postoperative day 3. Treatment with TREM2 markedly increased synaptophysin expression in both WT and transgenic mice compared with the gene-matched surgery group. ${ }^{*} \mathrm{P}<0.05$ versus the gene-matched control group; $\# \mathrm{P}<0.05$ versus the gene-matched surgery group. C: control group; $\mathrm{S}$ : surgery group; P3, P7, and P14: postoperative days 3, 7 , and 14 , respectively.

This study demonstrates that surgical trauma exacerbates behavioral deficits and enhances proinflammatory cytokine production in the APPswe/PS1dE9 (vs. WT) mice. Overexpression of TREM2 ameliorates surgery-induced neuroinflammatory responses by modulation of microglial function, which was accompanied by an improvement in cognitive function. TREM2 plays a protective role against tau pathology by inhibiting the activity of GSK-3 $\beta$. These data indicate that surgery-induced cognitive deficits may be mediated in part by upregulation of proinflammatory cytokines in the hippocampus. TREM 2 may represent a potential therapeutic target for surgery-induced neurobehavioral deficits.

Mounting evidence suggests that pre-existing AD pathology may be associated with the development of POCD [28]. A recent study by Murray et al. demonstrated that mice with pre-existing hippocampal neuronal damage were more vulnerable to hippocampal memory decline after an inflammatory challenge [29]. Accordingly, it has been shown that patients who have pre-existing neuronal damage are at higher risk of developing POCD [30, 31]. These data indicate that pre-existing neuronal damage may determine the vulnerability to the inflammation-mediated cognitive changes. In this study, exacerbated cognitive impairments and exaggerated neuroinflammatory responses were found in the transgenic 


\section{Cellular Physiology Cell Physiol Biochem 2018;46:1398-1411 \\ \begin{tabular}{c|c|c|c|} 
DOI: 10.1159/000489155 & O 2018 The Author(s). Published by S. Karger AG, Basel \\
www.karger.com/cpb
\end{tabular}}

Jiang et al.: TREM2 Ameliorates Postoperative Cognitive Deficits

surgery mice when compared with their WT counterparts. This susceptibility may be the result of pre-existing $\mathrm{AD}$ pathology that influences the vulnerability for surgery-induced neuroinflammatory process.

Mounting evidence has indicated that TREM2 plays a crucial role in cognitive performance and brain function [32]. TREM2 dysfunction has been shown to result in an autosomal recessive disorder called Nasu-Hakola disease, which is characterized by progressive presenile inflammatory neurodegeneration and severe cognitive impairment [33]. In addition, mutation-induced dysfunction of TREM2 has been shown to lead to memory deficits [34]. Furthermore, reduction of TREM2 expression contributes to the pathogenesis of AD. Overexpression of TREM2 in the brain of middle-aged APPswe/PS1dE9 mice could ameliorate AD-related neuropathology and improve spatial cognitive impairment [16]. In the present study, surgical trauma induced neurobehavioral deficits in both WT and transgenic mice. These behavioral disturbances were coupled with reduced TREM2 expression and exaggerated neuroinflammatory responses. Conversely, overexpression of TREM2 attenuated the behavioral deficits. Taken together, these data highlight a critical role of TREM2 in surgery-induced neurobehavioral deficits. However, it should be noted that our results did not support a direct involvement of TREM2 in spatial cognitive functions, as downregulation of TREM2 in the brain of WT mice was not associated with behavioral deficits in open-field and MWM tests on postoperative day 7. The protective effect of TREM2 is likely attributable to the suppression of neuroinflammation and subsequent attenuation of behavioral deficits.

TREM2 was revealed to regulate neuroinflammatory responses by repression of microglia-mediated cytokine production [12]. A study by Jiang et al. found that TREM2 overexpression reduced proinflammatory cytokine expression in a transgenic mouse model of AD [16]. TREM2 knockdown was shown to enhance IL- 6 and TNF- $\alpha$ expression in primary microglia or in the brain of SAMP8 mice [35]. Meanwhile, Wang and colleagues revealed that TREM2 deficiency enhanced neuroinflammatory responses in a 5XFAD mouse model of AD [11]. Additionally, anti-inflammatory cytokine IL-10 was markedly decreased by TREM2 deficiency [15]. This study provided more direct evidence that selectively overexpressed TREM2 markedly ameliorates surgery-induced neuroinflammatory responses and improves spatial cognitive impairment in these transgenic mice.

Microglia play a major role in the development of POCD [2, 7]. TREM2 is uniquely expressed on the microglia in the brain, supporting the view that TREM2 is a microglia-specific receptor [36]. Evidence shows that microglia participate in amyloid plaque compaction via a TREM2-dependent mechanism, forming a protective barrier that attenuates toxicity toward nearby neurons [16]. In this study, surgery-induced downregulation of TREM2 was accompanied by amplified microglial activation as indicated by increased Iba- 1 expression. Importantly, excessive microglial activation was improved by overexpression of TREM2. This finding indicates that surgery-induced dysfunction of TREM2 may lead to microglial activation, which subsequently caused cognitive deficiency.

Whether microglia provide a beneficial or detrimental effect on brain function has not been fully elucidated. On the basis of gene expression profiles, activated microglia may be categorized into two opposite types: M1 phenotype and M2 phenotype. The M1-like phenotype can result in the release of proinflammatory cytokines and subsequently lead to bystander damage of neurons [37, 38]. In contrast, the M2-like phenotype of microglia is characterized by an enhanced phagocytic activity as well as a reduced neuroinflammatory response $[37,38]$. In this study, treatment with TREM2 directly increased M2 phenotype marker Arg1 expression and inhibited microglia-mediated proinflammatory cytokine production following surgical challenge. TREM2 overexpression increased the levels of Arg1, implying that TREM2 suppresses neuroinflammatory responses through switching microglia toward the M2 phenotype.

Progression of tau pathology is directly associated with neuroinflammatory responses and loss of neurons and synapses [39]. Compelling evidence indicates that neuroinflammation could directly elevate the activity of tau kinases and thus contribute to tau pathology $[40,41]$. 


\section{Cellular Physiology Cell Physiol Biochem 2018;46:1398-1411

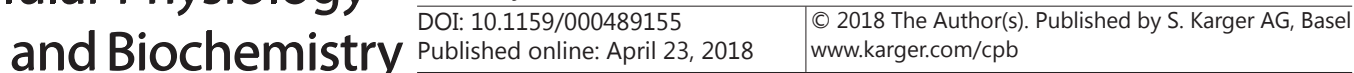

Jiang et al.: TREM2 Ameliorates Postoperative Cognitive Deficits

TREM2 was positively correlated with phosphorylated tau protein. Cruchaga and colleagues showed that the TREM2 R47H variant was related to higher levels of hyperphosphorylated tau protein in the cerebrospinal fluid of AD patients [42]. This finding has been confirmed by Lill et al. in a larger cohort of AD patients [43]. Intriguingly, silencing of microglial TREM2 in the brain of P301S mice, an animal model of tau pathology, markedly enhanced tau hyperphosphorylation and exacerbated spatial cognitive deficits [35]. Consistent with the previous study, surgical trauma enhanced tau hyperphosphorylation in the APPswe/PS1dE9 mice and treatment with TREM2 significantly ameliorated tau hyperphosphorylation in this study. These data strongly suggest that TREM2 might exert a protective effect in tau-related pathology.

Tau hyperphosphorylation usually results from the hyperactivation of tau kinase and/ or inactivation of tau phosphatases in the brain [44]. GSK-3 $\beta$ is a major kinase responsible for tau hyperphosphorylation. Surgical trauma significantly enhanced GSK-3 $\beta$ activity in the APPswe/PS1dE9 transgenic mice. More importantly, treatment with TREM2 dramatically inhibited GSK-3 $\beta$ activity and decreased tau hyperphosphorylation in this study. However, TREM2 overexpression failed to alter the activity of PP2A, the main phosphatase that participates in tau dephosphorylation. These findings imply that the protective effects of TREM2 against tau pathology are achieved, at least in part, by attenuating the activity of GSK-3 $\beta$.

Neuronal and synaptic integrity is required for maintenance of normal cognitive functions. Synaptophysin plays an important role in the regulation of synaptic plasticity. Mounting evidence indicates that microglia-generated proinflammatory cytokines (e.g., IL-6 and TNF- $\alpha$ ) are responsible for neuronal and synaptic losses, whereas anti-inflammatory therapies can effectively attenuate neuronal and synaptic damage and improve cognitive deficits in animal models of neuroinflammation and aging [45, 46]. TREM2 silencing led to the exacerbation of neuronal and synaptic losses in the cerebral cortex and hippocampus of P301S mice [47]. Surgery-induced downregulation of synaptophysin in the hippocampus contributed to spatial cognitive dysfunctions in this study. Overexpression of TREM2 attenuates synaptic losses and improves behavioral deficits in the APPswe/PS1dE9 mice [48].

It is noteworthy that the current study has some potential limitations. Only the strategy of adeno-associated virus vectors was employed to overexpress TREM2 in the brain of APPswe/PS1dE9 mice. This evidence may not be robust, owing to the individual variability in transduction efficiency. APPswe/PS1dE9 mice with TREM2 deficiency will be generated in future studies by genetic approaches to provide more direct evidence on the role of TREM2 in surgery-induced neuroinflammation and cognitive deficiency. It should be noted that lentiviral infection was well tolerated by animals, as no sign of neurotoxicity including hindlimb paralysis, vocalization, food intake, or neuroanatomical damage was observed during the entire study.

Inconclusion, TREM2 playsa pivotalroleininhibitingsurgery-inducedneuroinflammatory responses by modulation of microglial function and enhancing synaptophysin expression, which was accompanied by an improvement in cognitive function following a major surgical stress. TREM 2 also attenuates the activity of GSK- $3 \beta$, and thus plays a protective role against tau pathology. Taken together, these findings provide insights into the beneficial role of TREM2 in surgery-induced neuroinflammatory responses and neurobehavioral deficits and highlight TREM2 as a potential therapeutic target for the prevention/or treatment of POCD.

\section{Acknowledgements}

This work was funded by the National Natural Science Foundation of China (No. 81300938). 


\section{Cellular Physiology Cell Physiol Biochem 2018;46:1398-1411 \begin{tabular}{l|l} 
and Biochemistry Published online: April23, 2018 & $\begin{array}{l}\text { (c) } 2018 \text { The Author(s). Published by S. Karger AG, Basel } \\
\text { www.karger.com/cpb }\end{array}$
\end{tabular}}

Jiang et al.: TREM2 Ameliorates Postoperative Cognitive Deficits

\section{Disclosure Statement}

No conflict of interests exists.

\section{References}

1 Moller JT, Cluitmans P, Rasmussen LS, Houx P, Rasmussen H, Canet J, Rabbitt P, Jolles J, Larsen K, Hanning CD, Langeron O, Johnson T, Lauven PM, Kristensen PA, Biedler A, Beem HV, Fraidakis O, Silverstein JH, Beneken JEW,Gravenstein JS: Long-term postoperative cognitive dysfunction in the elderly ISPOCD1 study. ISPOCD investigators. International Study of Post-Operative Cognitive Dysfunction. Lancet 1998; 351:857861.

-2 Cao XZ, Ma H, Wang JK, Liu F, Wu BY, Tian AY, Wang LL, Tan WF: Postoperative cognitive deficits and neuroinflammation in the hippocampus triggered by surgical trauma are exacerbated in aged rats. Prog Neuropsychopharmacol Biol Psychiatry 2010;34:1426-1432.

3 Steinmetz J, Christensen KB, Lund T, Lohse N, Rasmussen LS: Long-term consequences of postoperative cognitive dysfunction. Anesthesiology 2009;110:548-555.

-4 Vasconcelos AR, Yshii LM, Viel TA, Buck HS, Mattson MP, Scavone C, Kawamoto EM: Intermittent fasting attenuates lipopolysaccharide-induced neuroinflammation and memory impairment. J Neuroinflammation DOI: 10.1186/1742-2094-11-85.

5 Zhang Z, Li X, Li F, An L: Berberine alleviates postoperative cognitive dysfunction by suppressing neuroinflammation in aged mice. Int Immunopharmacol 2016;38:426-433.

6 Hovens IB, Schoemaker RG, van der Zee EA, Absalom AR, Heineman E, van Leeuwen BL: Postoperative cognitive dysfunction: Involvement of neuroinflammation and neuronal functioning. Brain Behav Immun 2014;38:202-210.

7 Li Z, Liu F, Ma H, White PF, Yumul R, Jiang Y, Wang N, Cao X: Age exacerbates surgery-induced cognitive impairment and neuroinflammation in Sprague-Dawley rats: the role of IL-4. Brain Res 2017;1665:65-73.

-8 Ma Y, Cheng Q, Wang E, Li L, Zhang X: Inhibiting tumor necrosis factor-alpha signaling attenuates postoperative cognitive dysfunction in aged rats. Mol Med Rep 2015;12:3095-3100.

-9 Belarbi K, Jopson T, Tweedie D, Arellano C, Luo W, Greig NH, Rosi S: TNF-alpha protein synthesis inhibitor restores neuronal function and reverses cognitive deficits induced by chronic neuroinflammation. J Neuroinflammation DOI: 10.1186/1742-2094-9-23.

10 Kleinberger G, Yamanishi Y, Suárez-Calvet M, Czirr E, Lohmann E, Cuyvers E, Struyfs H, Pettkus N, Wenninger-Weinzierl A, Mazaheri F, Tahirovic S, Lleó A, Alcolea D, Fortea J, Willem M, Lammich S, Molinuevo JL, Sánchez-Valle R, Antonell A, Ramirez A, Heneka MT, Sleegers K, van der Zee J, Martin JJ, Engelborghs S, Demirtas-Tatlidede A, Zetterberg H, Van Broeckhoven C, Gurvit H, Wyss-Coray T, Hardy J, Colonna M, Haass C. TREM2 mutations implicated in neurodegeneration impair cell surface transport and phagocytosis. Sci Transl Med DOI:10.1126/scitranslmed.3009093.

11 Wang Y, Cella M, Mallinson K, Ulrich JD, Young KL, Robinette ML, Gilfillan S, Krishnan GM, Sudhakar S, Zinselmeyer BH, Holtzman DM, Cirrito JR, Colonna M:TREM2 lipid sensing sustains the microglial response in an Alzheimer's disease model. Cell 2015;160:1061-1071.

12 Hickman SE, El Khoury J: TREM2 and the neuroimmunology of Alzheimer's disease. Biochem Pharmacol 2014;88:495-498.

-13 Hsieh CL, Koike M, Spusta SC, Niemi EC, Yenari M, Nakamura MC, Seaman WE: A role for TREM2 ligands in the phagocytosis of apoptotic neuronal cells by microglia. J Neurochem 2009;109:1144-1156.

14 Takahashi K, Rochford CD, Neumann H: Clearance of apoptotic neurons without inflammation by microglial triggering receptor expressed on myeloid cells-2. J Exp Med 2005;201:647-657.

15 Jiang T, Yu JT, Zhu XC, Tan MS, Gu LZ, Zhang YD, Tan L: Triggering receptor expressed on myeloid cells 2 knockdown exacerbates aging-related neuroinflammation and cognitive deficiency in senescenceaccelerated mouse prone 8 mice. Neurobiol Aging 2014;35:1243-1251.

16 Jiang T, Tan L, Zhu XC, Zhang QQ Cao L, Tan MS, Gu LZ, Wang HF, Ding ZZ, Zhang YD, Yu JT: Upregulation of TREM2 ameliorates neuropathology and rescues spatial cognitive impairment in a transgenic mouse model of Alzheimer's disease. Neuropsychopharmacology 2014;39:2949-2962. 


\section{Cellular Physiology Cell Physiol Biochem 2018;46:1398-1411 \begin{tabular}{l|l} 
DOI: 10.1159/000489155 & Ond Biochemistry 2018 The Author(s). Published by S. Karger AG, Basel \\
wwww.karger.com/cpb
\end{tabular}

17 Huang TJ, Hsu RW, Li YY, Cheng CC: Less systemic cytokine response in patients following microendoscopic versus open lumbar discectomy. J Orthop Res 2005;23:406-411.

18 Nelson CJ, Lysle DT: Severity, time, and beta-adrenergic receptor involvement in surgery-induced immune alterations. J Surg Res 1998;80:115-122.

19 Canet J, Raeder J, Rasmussen LS, Enlund M, Kuipers HM, Hanning CD, Jolles J, Korttila K, Siersma VD, Dodds C, Abildstrom H, Sneyd JR, Vila P, Johnson T, Muñoz Corsini L, Silverstein JH, Nielsen IK, Moller JT; ISPOCD2 investigators: Cognitive dysfunction after minor surgery in the elderly. Acta Anaesthesiol Scand 2003; 47:1204-1210.

20 Rosczyk HA, Sparkman NL, Johnson RW: Neuroinflammation and cognitive function in aged mice following minor surgery. Exp Gerontol 2008;43:840-846.

21 Wang N, Ma H, Li Z, Gao Y, Cao X, Jiang Y, Zhou Y, Liu S: Chronic unpredictable stress exacerbates surgeryinduced sickness behavior and neuroinflammatory responses via glucocorticoids secretion in adult rats. PloS one DOI: 10.1371/journal.pone.0183077.

-22 Tian Y, Guo S, Zhang Y, Xu Y, Zhao P, Zhao X: Effects of Hydrogen-Rich Saline on Hepatectomy-Induced Postoperative Cognitive Dysfunction in Old Mice. Mol Neurobiol 2017;54:2579-2584.

-23 Li M, Yong-Zhe L, Ya-Qun M, Sheng-Suo Z, Li-Tao Z, Ning-Ling P: Ulinastatin alleviates neuroinflammation but fails to improve cognitive function in aged rats following partial hepatectomy. Neurochem Res 2013;38:1070-1077.

24 Ruth MB, Amy MH, Matthew GF, Linda RW, Steven FM: Intracisternal interleukin-1 receptor antagonist (IL1RA) prevents post-operative cognitive decline and neuroinflammatory response in aged rats. J Neurosci 2012;32:14641-14648.

25 Le Y, Liu S, Peng M, Tan C, Liao Q, Duan K, Ouyang W, Tong J: Aging differentially affects the loss of neuronal dendritic spine, neuroinflammation and memory impairment at rats after surgery. PloS one DOI: 10.1371/ journal.pone.0106837.

-26 Kawabori M, Kacimi R, Kauppinen T, Calosing C, Kim JY, Hsieh CL, Nakamura MC, Yenari MA: Triggering receptor expressed on myeloid cells 2 (TREM2) deficiency attenuates phagocytic activities of microglia and exacerbates ischemic damage in experimental stroke. J Neurosci 2015;35:3384-3396.

27 Zhong L, Chen XF, Zhang ZL, Wang Z, Shi XZ, Xu K, Zhang YW, Xu H, Bu G: DAP12 Stabilizes the C-terminal Fragment of the Triggering Receptor Expressed on Myeloid Cells-2 (TREM2) and Protects against LPSinduced Pro-inflammatory Response. J Biol Chem 2015; 290:15866-15877.

28 Evered L, Silbert B, Scott DA, Ames D, Maruff P, Blennow K: Cerebrospinal Fluid Biomarker for Alzheimer Disease Predicts Postoperative Cognitive Dysfunction. Anesthesiology 2016;124:353-361.

29 Murray C, Sanderson DJ, Barkus C, Deacon RM, Rawlins JN, Bannerman DM, Cunningham C: Systemic inflammation induces acute working memory deficits in the primed brain: relevance for delirium. Neurobiol Aging 2012;33:603-616.

-30 Hudetz JA, Patterson KM, Amole O, Riley AV, Pagel PS: Postoperative cognitive dysfunction after noncardiac surgery: effects of metabolic syndrome. J Anesth 2011;25:337-344.

-31 Bekker A, Lee C, de Santi S, Pirraglia E, Zaslavsky A, Farber S, Haile M, de Leon MJ: Does mild cognitive impairment increase the risk of developing postoperative cognitive dysfunction? Am J Surg 2010;199:782788.

-32 Jiang T, Yu JT, Zhu XC, Tan L: TREM2 in Alzheimer's disease. Mol Neurobiol 2013;48:180-185.

-33 Sasaki A, Kakita A, Yoshida K, Konno T, Ikeuchi T, Hayashi S, Matsuo H, Shioda K: Variable expression of microglial DAP12 and TREM2 genes in Nasu-Hakola disease. Neurogenetics 2015;16:265-276.

-34 Montalbetti L, Ratti MT, Greco B, Aprile C, Moglia A, Soragna D: Neuropsychological tests and functional nuclear neuroimaging provide evidence of subclinical impairment in Nasu-Hakola disease heterozygotes. Funct Neurol 2005;20:71-75.

-35 Jiang T, Zhang YD, Chen Q Gao Q, Zhu XC, Zhou JS, Shi JQ, Lu H, Tan L, Yu JT: TREM2 modifies microglial phenotype and provides neuroprotection in P301S tau transgenic mice. Neuropharmacology 2016;105:196-206.

-36 Srinivasan K, Friedman BA, Larson JL, Lauffer BE, Goldstein LD, Appling LL, Borneo J, Poon C, Ho T, Cai F, Steiner P, van der Brug MP, Modrusan Z, Kaminker JS, Hansen DV: Untangling the brain's neuroinflammatory and neurodegenerative transcriptional responses. Nat Commun DOI: 10.1038/ ncomms11295. 


\section{Cellular Physiology Cell Physiol Biochem 2018;46:1398-1411 \begin{tabular}{l|l} 
DOI: 10.1159/000489155 & O 2018 The Author(s). Published by S. Karger AG, Basel \\
www.karger.com/cpb
\end{tabular}}

Jiang et al.: TREM2 Ameliorates Postoperative Cognitive Deficits

-37 Olah M, Biber K, Vinet J, Boddeke HW: Microglia phenotype diversity. CNS Neurol Disord Drug Targets 2011;10:108-118.

-38 Patel AR, Ritzel R, McCullough LD, Liu F: Microglia and ischemic stroke: a double-edged sword. Int J Physiol Pathophysiol Pharmacol 2013;5:73-90.

-39 Frost B, Gotz J, Feany MB: Connecting the dots between tau dysfunction and neurodegeneration. Trends Cell Biol 2015;25:46-53.

40 Ghosh S, Wu MD, Shaftel SS, Kyrkanides S, LaFerla FM, Olschowka JA, O'Banion MK: Sustained interleukin1 beta overexpression exacerbates tau pathology despite reduced amyloid burden in an Alzheimer's mouse model. J Neurosci 2013;33:5053-5064.

41 Gorlovoy P, Larionov S, Pham TT, Neumann H: Accumulation of tau induced in neurites by microglial proinflammatory mediators. FASEB J 2009;23:2502-2513.

42 Cruchaga C, Kauwe JS, Harari O, Jin SC, Cai Y, Karch CM, Benitez BA, Jeng AT, Skorupa T, Carrell D, Bertelsen S, Bailey M, McKean D, Shulman JM, De Jager PL, Chibnik L, Bennett DA, Arnold SE, Harold D, Sims R, Gerrish A, Williams J, Van Deerlin VM, Lee VM, Shaw LM, Trojanowski JQ Haines JL, Mayeux R, PericakVance MA, Farrer LA, Schellenberg GD, Peskind ER, Galasko D, Fagan AM, Holtzman DM, Morris JC; GERAD Consortium; Alzheimer's Disease Neuroimaging Initiative (ADNI); Alzheimer Disease Genetic Consortium (ADGC), Goate AM: GWAS of cerebrospinal fluid tau levels identifies risk variants for Alzheimer's disease. Neuron 2013;78:256-268.

43 Lill CM, Rengmark A, Pihlstrom L, Fogh I, Shatunov A, Sleiman PM, Wang LS, Liu T, Lassen CF, Meissner E,Alexopoulos P, Calvo A, Chio A, Dizdar N, Faltraco F, Forsgren L, Kirchheiner J, Kurz A, Larsen JP, Liebsch M, Linder J, Morrison KE, Nissbrandt H, Otto M, Pahnke J, Partch A, Restagno G, Rujescu D, Schnack C, Shaw CE, Shaw PJ, Tumani H, Tysnes OB, Valladares O, Silani V, van den Berg LH, van Rheenen W, Veldink JH, Lindenberger U, Steinhagen-Thiessen E; SLAGEN Consortium, Teipel S, Perneczky R, Hakonarson H, Hampel H, von Arnim CAF, Olsen JH, Van Deerlin VM, Al-Chalabi A, Toft M, Ritz B, Bertram L: The role of TREM2 R47H as a risk factor for Alzheimer's disease, frontotemporal lobar degeneration, amyotrophic lateral sclerosis, and Parkinson's disease. Alzheimers Dement 2015;11:1407-1416.

44 Tao G, Zhang J, Zhang L, Dong Y, Yu B, Crosby G, Culley DJ, Zhang Y, Xie Z: Sevoflurane induces tau phosphorylation and glycogen synthase kinase 3 beta activation in young mice. Anesthesiology 2014;121(3):510-527.

45 Jin P, Kim JA, Choi DY, Lee YJ, Jung HS, Hong JT: Anti-inflammatory and anti-amyloidogenic effects of a small molecule, 2, 4-bis(p-hydroxyphenyl)-2-butenal in Tg2576 Alzheimer's disease mice model. J Neuroinflammation DOI: 10.1186/1742-2094-10-2.

-46 Lee YJ, Choi DY, Choi IS, Kim KH, Kim YH, Kim HM, Lee K, Cho WG, Jung JK, Han SB, Han JY, Nam SY, Yun YW, Jeong JH, Oh KW, Hong JT: Inhibitory effect of 4-O-methylhonokiol on lipopolysaccharide-induced neuroinflammation, amyloidogenesis and memory impairment via inhibition of nuclear factor-kappaB in vitro and in vivo models. J Neuroinflammation DOI: 10.1186/1742-2094-9-35.

47 Jiang T, Tan L, Zhu XC, Zhou JS, Cao L, Tan MS, Wang HF, Chen Q, Zhang YD, Yu JT: Silencing of TREM2 exacerbates tau pathology, neurodegenerative changes, and spatial learning deficits in P301S tau transgenic mice. Neurobiol Aging 2015;36:3176-3186.

-48 Martin L, Latypova X, Wilson CM, Magnaudeix A, Perrin ML, Terro F: Tau protein phosphatases in Alzheimer's disease: the leading role of PP2A. Ageing Res Rev 2013;12:39-49. 